\title{
Mediating role of resilience between successful aging and acculturation among older Korean immigrants In the United States
}

\author{
Jaewon Lee, PhD, MSW \\ Department of Social Work \\ California State University Bakersfield
}

\begin{abstract}
Background: This study was to investigate how acculturative stress affects older Korean immigrants' successful aging and whether resilience can play a mediator's role to the sample population's successful aging or not. The research design was a cross-sectional survey using three valid and reliable scales such as Successful Aging Scale, Acculturative Stress Scale, and Resilience Scale. A research shows that there is no or minimum correlation between acculturative stress and successful aging (Choi \& Thomas, 2009). However, it was hypothesized that although there was a weak correlation between acculturative stress and successful aging, once the resilience involved as a mediator, the correlation would be stronger.

Methods: The study was designed as a self-administered survey research using the scales mentioned above. One hundred and two $(\mathrm{N}=102)$ older Koreans who currently enrolled in Adult Health Care Centers in Los Angeles, CA, participated in this study. Multiple Regression, Correlation, ANOVA, and $t$-test was administered to analyze the collected data.

Results: The results indicated that there was a positive but weak correlation between acculturative stress and the sample population's successful aging rate $(r=.252, \mathrm{p}<.05)$. Interestingly, when resilience intervened as a mediator, the correlation between acculturation and successful aging was increased to $(r=.622, p<.05)$. Therefore, the study discovered that the resilience was able to play a mediator's role between acculturative stress and successful aging among the older Korean immigrants.

Implications: The findings can be directed to social work researchers and practitioners to help educate the ethnic older adults in the community on the social and behavioral importance of the positive aspects of aging such as successful aging and healthy aging to increase the chance of community member's longevity. Future research will include other ethnic and immigrant older populations in the community to show internal and external validity of the study related to successful aging, acculturative stress, and resilience.
\end{abstract}


Lee, J. (2020). Mediating role of resilience between successful aging and acculturation among older Korean immigrants In the United States. Advances in Social Sciences Research Journal, 7(5) 117-133.

\section{INTRODUCTION}

In 2017, the older population in the United States was 40 million and is expected to be about 78 million by 2035 (US Census Bureau, 2018). According to a statistical report from World Population Aging (2017), the older population will account for approximately $25 \%$ of the total world's population by 2035 . When $20 \%$ or more of the total population is 65 or older, the society can be called a "super aging society" (Muramatsu \& Akiyama, 2011; Ortman, Velkoff, \& Hogan, 2014).

Once a society turns into super aging society, it will meet new problems in social, economic, and cultural dimensions (Ortman, Velkoff, \& Hogan, 2014; Muramatsu \& Akiyama, 2011). It will need to improve our social policies for the rapidly increasing older population, and governmental expenses for the older population should be reconsidered because of the considerably higher cost of medical and long-term care services needed. In our communities, more facilities and social infrastructure for Senior Citizens' leisure and their retirement life will be needed as well, and this might change our culture that has been focused on the younger generations (Ortman, Velkoff, \& Hogan, 2014; Muramatsu \& Akiyama, 2011).

Approximately 2.7 million Korean immigrants reside in the United States (US Census Bureau, 2017), and the number is constantly growing. In 2020, the estimated population of Koreans who are 65 or older is expected about $20-25 \%$ of the total population (Choi \& Thomas, 2009). In the case of metropolitan areas such as Los Angeles and New York City, the ratio of the Korean older population is estimated at up to $30 \%$ of the total Korean immigrant population. Today, older Korean immigrants have become an important social work client group because studies show higher ratio of the population place under marginalized areas than any other immigrant populations in the United States (August \& Sorkin, 2010; Hooyman \& Kiyak, 2011).

\section{Purpose of Study}

The purpose of this study is to measure the rate of successful aging, resilience, and acculturation among the population of older Korean immigrants in the United States and to examine the relationships. Measuring the level of successful aging, resilience, and acculturation, and investigating their relationships is important in terms of providing a better understanding of older Korean immigrants' actual status of later life such as health status, social engagement, supportive systems, coping mechanisms, and the process of adaptation to new environments as immigrants. Since older Korean immigrants have a higher probability of marginalization due to acculturative stress, lower socioeconomic status, and health care disparities (August \& Sorkin, 2010; Hooyman \& Kiyak, 2011), the relationship with successful aging, resilience, and acculturation can play an important role in determining the rate of successful aging among the population.

\section{Successful Aging}

\section{LITERATURE REVIEW}

Many studies have focused on how to age well (Atchley, 1989; Baltes \& Baltes, 1990; Cumming \& Henry, 1961; Havighurst 1961; Larson \& Phelan, 2001; Li et al., 2006; Liang \& Luo, 2012; Rowe \& Kahn, 1997). Among those studies, Rowe and Kahn's Successful Aging has been assessed as one of the best-conceptualized healthy aging studies over the past decade (Chung \& Park, 2008; Depp \& Jeste, 2006; Ferri, James, \& Pruchno, 2009). It has been considered one of the representative studies on successful aging, and it has been one of the most influential studies on aging in the field of 
Gerontology (Depp \& Jeste, 2006; Ferri, James, \& Pruchno, 2009; Maki, 2005; Nagalingam, 2007; Victor, 2004).

Rowe and Kahn (1997) defined successful aging as "an avoidance of disease and disability, maintenance of high physical and cognitive function, and engagement with social and productive activities" (p.439). If an older adult displayed no or few diseases and health-related disabilities, maintained high physical and cognitive functions, and sustained social and productive activities, we could call the older adult a successful ager by the definition of Rowe and Kahn's theory (Ferri, James, \& Pruchno, 2009).

Numerous studies (Chung \& Park, 2008; Cernin, 2009; Tate, Lah, \& Cuddy, 2003; Larson \& Phelan, 2001; Li et al., 2006; Liang \& Luo, 2012; Maki, 2005; Nagalingam, 2007; Victor, 2004) have employed the theories of successful aging as their main conceptual framework in gerontological research studies in the United States and other countries such as South Korea, China, Singapore, and some European and Latin American countries. These studies in other countries also have displayed some commonalities in the components of successful aging, indicating that these ideas can be important factors in determining if the older minority populations in the United States are successful agers or not.

A study about successful aging among low-income older adults was conducted by Chung and Park (2008) in South Korea. In this qualitative research study, the researchers interviewed 220 older South Korean adults. According to the researchers, the low-income older population in South Korea defines successful as being satisfied in old age with one's quality of life and being able to adapt successfully to various changes and losses that accompany the aging process. The most important factor for maintaining successful aging among the population was that successful aging can be achieved when a person is satisfied with his/her later life because they consider success as independent of social or economic status.

The study also found that the population was built on strong relationships with others and a positive attitude such as resilience toward life for a successful old age. A significant finding was that the important conditions of successful aging is the success of adult children. Their adult children's educational, financial, and social achievements are very important factors in determining their rate of successful aging and it is a motivation to overcome their negative situations such as financial hardship or deterioration of health (Chung \& Park, 2008).

\section{Resilience}

Resilience theory was used in the current study to examine the relationship between successful aging and resilience among the population. Resilience is the ability to bounce back and the willingness to overcome negative influences that block achievement. It is the ability to thrive in the face of adversity and to adapt body, mind, and spirit to current life circumstances (Richardson, 2002). Based on my literature review, the resilience theory has been used in most cases to support research studies to examine children's ability to overcome their negative circumstances such as domestic violence, diseases, post war, or natural disaster. However, some studies (Fuller-Iglesias, Sellars, \& Antonucci, 2008; Jeste et al, 2013; Landau, 2007; Martin, Distelberg, \& Elahad, 2015; Smith \& Hayslip, 2012; Resnick, 2010; Resnick \& Inguito, 2011) indicate that this concept could be used to study the older population's ability to overcome their negative environment such as deteriorated 
Lee, J. (2020). Mediating role of resilience between successful aging and acculturation among older Korean immigrants In the United States. Advances in Social Sciences Research Journal, 7(5) 117-133.

health conditions, lower socio-economic status, cultural differences, and language barriers if older adults were aware of the concept and were able to apply the concept to their lives.

The key concepts for understanding the process of resilience are competence, adversity, assets and risk, and protective processes and vulnerabilities (Conner \& Davidson, 2003). Resilience theory (Richardson, 2002) states that if a person were faced with stressors, adversity, and unexpected life events, he/she would interact with previous experiences of disruptions and be ready for reintegration. Then, he/she would be willing to reintegrate resiliently and return to homeostasis, which is the status of biopsychospiritual balance.

Measuring resilience is important as it helps show how older immigrants adapt to challenging environments (Lavretsky, 2012; Resnick \& Inguito, 2011; Yee-Melichar, 2011). The concept of resilience will be applied to older minorities/immigrants at higher risk of marginalization, having disease and disability, impoverishment, isolation, discrimination, and any other limitations due to lower socioeconomic status. Older minorities/immigrants are double- or triple-jeopardized to be marginalized due to lower socioeconomic status, health care disparities, and acculturative stress (Hooyman \& Kiyak, 2011). Thus, resilience can be an essential element for successful aging (Martin, Distelberg, \& Elahad, 2015) for older ethnic minorities such as older Korean immigrants who have been suffering from a higher level of psychological distress, functional limitations, lower socioeconomic status, and acculturative stress.

In the case of older Korean immigrants, who are certain to confront adversities and experience extra biopsychosocial problems resulting from acculturative stress in addition to their normal aging process, they will be able to age successfully if they can access resilient assets such as protective processes, self-efficacy, supportive networks, and community engagement (Hardy, Concato, \& Gill, 2004). They would likely overcome the adversities such as language barriers, lower socio-economic status if they were provided with more protective processes and resilient assets with those systematic supports (Conner \& Davidson, 2003; Richardson, 2002; Hooyman \& Kiyak, 2011).

\section{Acculturation}

Acculturation has been defined as the process of change or adaptation in one's attitudes, values, behavior, and identity that results from experiencing a new cultural context (Kim, Kim, \& Gulick, 2009; Park \& Bernstein, 2008; Berry, 1997). Previous studies have examined various components of acculturation including language proficiency, religious activities, national or cultural identification, food and media preferences, and attachment to cultural values and traditions (Oh et al., 2002; Jang, Kim, \& Chiriboga, 2005). Especially for older Korean immigrants who have a higher risk of being marginalized compared to other ethnic groups due to relatively lower socio-economic status, limited access to health and social services, and being vulnerable to social and linguistic isolation (Jang, Kim, \& Chiriboga, 2005; Min, Moon, \& Lubben, 2005; Lee, Moon, \& Knight, 2004; Mui, 2001), acculturation refers to a greater affiliation to the dominant social, cultural, and behavioral norms of the United States (Chae \& Foley, 2010; Finch \& Vega, 2003; Nguyen, 2011).

The acculturation model was used as the framework to undergird the sense of acculturation among the older Korean immigrants. According to Berry (2003), acculturation can affect the quality of life among immigrant populations in terms of increasing familiarity to mainstream culture and language and reducing acculturative stress. Therefore, it would be helpful to see how acculturated 
older immigrants remain resilient in negative environments. The acculturation model describes several options that older ethnic minorities can choose: assimilation, separation, integration, and marginalization (Berry, 2003).

According to the model, an older ethnic minority can be assimilated into the majority culture or they can be separated by a defensive assertion of the minority culture. They can be integrated by a blending of the two cultures and a cultural alternation between cultures, or they can be marginalized by a diminishment of both cultures. Hence, older Korean immigrants might be able to choose an option that implies that they still can be identified as a member of the minority culture even if they become a competent participant of the majority culture (Jang, Kim, \& Chiriboga, 2005; Jang, Kim, Chiriboga, King-Kallimanis, 2007; Rudmin, 2003).

Previous studies have shown that the level of acculturation can be representative of socio-economic status and an indication of social adaptation for older minorities and immigrants (Chiriboga \& Jang, 2010; Jang et al., 2006; Lee et al., 2000). According to the studies, those who are more acculturated tend to have better physical and mental health that can be positively linked to the level of successful aging compared to less acculturated ones. Older immigrants who are more acculturated to a new culture may have better adjustment to their situations and perceive their own aging processes in a more positive manner (Chriboga et al., 2002; Jang et al., 2006; Lee, Sobal, \& Fronglillo, 2000; Myers \& Rodriguez, 2002). In the current study, this model is applied to the population of older Korean immigrants to examine the relationship between resilience and successful aging among the population with acculturative stress.

\section{RESEARCH QUESTIONS AND HYPOTHESES}

Based on the literature review, as discussed above, the following research questions and hypotheses guided this study:

- Question 1. How does resilience influence successful aging among older Korean immigrants in the United States?

- Question 2. How does acculturation affect successful aging among older Korean immigrants in the United States?

- Hypothesis 1. A significant positive relationship exists between resilience and successful aging among the older Korean immigrants in the United States.

- Hypothesis 2. A significant relationship exists between acculturation and successful aging among the older Korean immigrants in the United States.

\section{Research Design}

\section{STUDY METHOD}

This study was designed as a self-administered survey research project using 1) the Successful Aging Scale, 2) the Resilience Scale, and 3) the Acculturation Scale. This study was a cross-sectional survey designed for descriptive and explanatory purposes. One hundred and two participants $(\mathrm{N}=102)$ who were attending two different Adult Day Health Care Centers in Los Angeles, California, participated in the cross-sectional survey. The survey was voluntary, and the informed consent form that was approved by the IRB institute was given to all the participants. The purpose of the 
Lee, J. (2020). Mediating role of resilience between successful aging and acculturation among older Korean immigrants In the United States. Advances in Social Sciences Research Journal, 7(5) 117-133.

study and detailed information about the scales were explained to the participants prior to the survey.

\section{Scales}

For this study, three pre-existing self-administered questionnaires (the Successful Aging Scale, the Resilience Scale, and the Acculturation Scale), and a demographic questionnaire including two open-ended questions were utilized for the participants in the United States. Since both the English version and the Korean version were available for the three pre-existing scales, the researcher needed to translate the questions in the demographic section only. The original version of the Successful Aging Scale has 14 items, but 10 items were selected for this study. In the case of the Resilience Scale, the original version has 25 items, but 20 items were chosen. All 20 items of the original Acculturation Scale were used. The questions in the demographic section were developed in English after scholarly review and then translated into a Korean version. Both versions were interpreted by two Certified translators.

The Successful Aging scale demonstrated relatively good psychometric properties such as a good internal consistency for the overall scores (Cronbach's alpha $=.84$ ) and scores ranged from 0-70 (5 points were given per each question). The high scorers on the Successful Aging Scale were more satisfied with their aging, and they reported a lower level of acculturative stress and a higher level of social engagement and physical and cognitive function. The Resilience Scale demonstrated relatively good psychometric properties such as a good internal consistency for the overall scores (Cronbach's alpha $=.89$ ), ranging range from 0-50 (5 points per question). The high scorers on the Resilience Scale showed greater satisfaction with their aging and reported a lower level of stress in their lives and a higher level of ability to bounce back and willingness to overcome their negative environments.

\section{Data Collection}

The participants were older Korean immigrants in the United States who were 65 years old or more, regardless of having specific physical diseases or disabilities, gender, marital status, income, occupation, and living with someone or living alone. Initially, the executive directors of the two Adult Day Health Care Centers were asked to participate in this study. Follow-up contacts with the administrators of the centers were made and both authorized program directors agreed to join this study and recruit voluntary participants to take the survey. Contacts for visiting the agencies were made with the program directors of the agencies, and the arrangements were made for in-person visits with verbal consent. One hundred percent of onsite surveys were administered in the agencies.

\section{Data Analysis}

The data analysis included consideration of demographic variables such as age, gender, and marital status. Due to the homogeneity of the Korean population, ethnicity was excluded from the demographic variables to be considered. Data was analyzed by descriptive analysis, coefficient (Cronbach's alpha), correlation (Pearson's r), simple regression, and multivariate regression analysis, using SPSS 20th version. Descriptive analysis describes the characteristics of the subjects as well as demographic information. Coefficient (Cronbach's alpha) tests the reliability of the measurement scales. To examine the relationship between successful aging and resilience among the sample population, a correlation analysis was used to determine whether there were significant 
relationships among the variables such as the scores of Successful Aging Scale and Resilience Scale. In addition, Pearson's $r$ was used for analysis of the correlation among other demographic variables.

\section{RESULTS}

A total of 102 Korean older adults who resided at the Korea Town in Los Angeles, California in the United States participated in this study. The sample ( $=102)$ was recruited in Los Angeles, $\mathrm{CA}$, and consisted of $38.2 \%(n=39)$ males and $61.8 \%(n=63)$ females. The average age of the participants was 83.49 years old, and the oldest respondent was 95 years old. A total of $38.2 \%(n=39)$ of the participants were married and $51.0 \%(n=52)$ were widowed. Regarding the sample's education, $36.3 \%(n=37)$ of the sample had received high school education or more, and $49.0 \%(n=50)$ had received elementary school education or less.

None of the participants answered that they currently had an occupation, and none of the participants answered that they were involved in volunteer work. Only one participant responded that currently he/she had a financial hardship, and one participant answered that he/she lacked money for groceries; in addition, $96.1 \%(n=98)$ of the participants answered that currently they had a certain type of income (SSI).

It was interesting that even though such a high number of respondents were receiving Supplemental Security Income, which supports people with very low or no income who are over 65 or blind or otherwise disabled (Social Security, 2014), and 3.9\% of the respondents did not have any type of financial resource, only one respondent answered 'Yes' to the question "Do you have financial hardship?" Based on verbal reports of the participants, the majority complained about their sparse financial resources. Perhaps they needed some extra money to maintain their dignity in their family and among friends because SSI was good enough to pay for their rent and groceries but not good enough to pay for their miscellaneous goods. This could be interpreted as if by receiving SSI, they considered themselves as people with income. Even if they were placed beyond the line of extreme poverty, not everyone understood the meaning of the benefit that they were receiving, and they felt they should appreciate what they had rather than complain about what they did not have. They just were being thankful to the American government for the benefit, but also anxious about the possibility of the benefit ending for any reason.

Of the total participants, 34.3\% $(n=35)$ lived with their spouse, $46.1 \%(n=47)$ lived alone, and 6.9\% $(n=7)$ lived with their children. A total of $53.9 \%(n=55)$ lived with someone (e.g. assistant). About their residence, $63.7 \%(n=65)$ of the participants lived in senior citizens' housing, 20.6\% $(n=21)$ rented their apartments, $12.7 \%(n=13)$ resided in a nursing home, and $2.9 \%(n=3)$ own their house or condo.

In the cases of self-rated health status, $42.2 \%(n=43)$ of the participants responded that their physical health was "good," 53.9\% $(n=55)$ responded that their emotional status was "good," and $54.9 \%(n=56)$ responded that their memory status was "good." Others rated their own health status as "fair": $37.3 \%(n=38)$, physical health; $23.5 \%(n=24)$, emotional status, and $27.5 \%(n=28)$, memory status. 
Lee, J. (2020). Mediating role of resilience between successful aging and acculturation among older Korean immigrants In the United States. Advances in Social Sciences Research Journal, 7(5) 117-133.

\section{Correlational Analysis}

Correlations were computed between the dependent variable (DV), successful aging, and one of the independent variables (IV), resilience, and another IV, acculturation. Table 1 presents correlations, whether positive or negative, as well as how strongly the pair of variables were related or not related. The results revealed a statistically significant positive relationship between the DV (successful aging) and one of the IV, resilience $(r=.622, p<.001)$ and a statistically significant positive relationship with another IV, acculturation $(r=.252, p<.01)$.

Table 1: Correlations of Successful Aging and Resilience and Acculturation

\begin{tabular}{|c|c|c|c|}
\hline Variable & & Resilience & Acculturation \\
\hline \multirow{2}{*}{ Successful Aging } & Pearson's $\boldsymbol{r}$ & $r=.622^{* *}$ & $r=.252^{*}$ \\
\cline { 2 - 4 } & $\boldsymbol{p}$ value & $p=.000$ & $p=.005$ \\
\hline
\end{tabular}

${ }^{* *}$ Correlation is significant at the 0.001 level (2-tailed).

*Correlation is significant at the 0.05 level (2-tailed).

Correlational analyses were performed to examine the relationship between successful aging and resilience, and the relationship between successful aging and acculturation. The results indicated that older Korean immigrants who reported a higher level of resilience and acculturation were more likely aging successfully. This suggests that older Korean immigrants who have more ability to bounce back in negative environments have greater chances to become successful agers. In addition, older Korean immigrants who have been more acculturated to American culture and language have greater probabilities to become successful agers.

\section{Regression Analysis}

Simple Linear Regression and Multiple Regression Analyses were conducted to test the relationship between successful aging and resilience, and the relationship between successful aging and acculturation. With regression analyses, the linearity, multivariate normality, and constant variance (homoscedasticity) among the variables were examined via visual inspection of linear regression plots. Figure 1 illustrates the linear regression plots and the results of such examinations. The plots constructed a shape of rectangular distribution of scores clustering in the center. This pattern also satisfied parametric assumptions (Mertler \& Vannatta, 2005).

Table 2 shows the Model Summary of the regression analysis, and Table 3 displays that resilience and acculturation were both significant and able to explain 38.8\% (Adjusted $R$ Square was .388) of the variance in successful aging $(F[2,99]=32.992, p<.001)$.

Table 2: The Model Summary of Successful Aging and Resilience and Acculturation

\begin{tabular}{|c|c|c|c|c|}
\hline Model & $\mathbf{R}$ & R Square & $\begin{array}{c}\text { Adjusted R } \\
\text { Square }\end{array}$ & $\begin{array}{c}\text { Std. Error of the } \\
\text { Estimate }\end{array}$ \\
\hline 1 & $.635^{*}$ & .400 & .388 & 5.108 \\
\hline
\end{tabular}

* Predictors (Constant), Resilience, Acculturation 
Table 3: Regression of Successful Aging and Resilience and Acculturation

\begin{tabular}{|c|c|c|c|c|c|c|}
\hline \multicolumn{2}{|c|}{ Model } & $\begin{array}{c}\text { Sum of } \\
\text { Squares }\end{array}$ & df & $\begin{array}{c}\text { Mean } \\
\text { Square }\end{array}$ & F & Sig. \\
\hline 1 & Regression & 1721.958 & 2 & 860.979 & 32.992 & $.000^{* *}$ \\
& Residual & 2583.532 & 99 & 26.096 & & \\
& Total & 4305.490 & 101 & & & \\
& & & & & & \\
\hline
\end{tabular}

*Dependent Variable: Successful Aging

**Predictors: (Constant), Resilience, Acculturation

Table 4 displays that the positive regression coefficient $(B=.153, \beta=.252, t[99]=10.990, p<.05)$ for acculturation indicated that a statistical significant relationship between successful aging and acculturation. This confirmed that the participants who had a higher level of acculturation were more likely to have a greater level of successful aging.

Table 4:Coefficients of Acculturation in Simple Linear Regression Analysis (N=102)

$* p<.05$

\begin{tabular}{|c|c|c|c|c|c|c|}
\hline \multicolumn{2}{|c|}{ Model } & \multicolumn{2}{|c|}{$\begin{array}{c}\text { Unstandardized } \\
\text { Coefficients }\end{array}$} & $\begin{array}{c}\text { Standardized } \\
\text { Coefficients }\end{array}$ & T & Sig. \\
\hline \multicolumn{2}{|c|}{} & B & $\begin{array}{c}\text { Std. } \\
\text { Error }\end{array}$ & Beta & & \\
\hline 1 & Constant & 28.583 & 2.601 & & 10.990 & .000 \\
\hline & $\begin{array}{c}\text { Acculturatio } \\
\text { n }\end{array}$ & .153 & .059 & .252 & 2.605 & $.011^{*}$ \\
\hline
\end{tabular}

Table 5 illustrates that the positive regression coefficient $(B=.153, \beta=.252, t[99]=10.990, p<.05)$ for resilience indicated that a statistical significant relationship between successful aging and resilience. This confirmed that the participants who had a higher level of resilience were more likely to have a greater level of successful aging.

Table 5:Coefficients of Resilience and Acculturation in Regression Analysis ( $\mathrm{N}=102)$

\begin{tabular}{|c|c|c|c|c|c|c|}
\hline \multicolumn{2}{|r|}{ Model } & \multicolumn{2}{|c|}{$\begin{array}{l}\text { Unstandardized } \\
\text { Coefficients }\end{array}$} & \multirow{2}{*}{$\begin{array}{c}\begin{array}{c}\text { Standardized } \\
\text { Coefficients }\end{array} \\
\text { Beta }\end{array}$} & \multirow[t]{2}{*}{$\mathbf{T}$} & \multirow[t]{2}{*}{ Sig. } \\
\hline & & B & $\begin{array}{l}\text { Std. } \\
\text { Error }\end{array}$ & & & \\
\hline 1 & Constant & 12.823 & 2.975 & & 4.309 & .000 \\
\hline & Resilience & .253 & .034 & .595 & 7.450 & .000 \\
\hline & $\begin{array}{c}\text { Acculturatio } \\
\mathrm{n}\end{array}$ & .073 & .049 & .119 & 1.495 & $.138^{*}$ \\
\hline
\end{tabular}

a. Dependent variable: Successful aging; Independent variables: Resilience and Acculturation

b. Model 1: $R=.632, R$ Square $=.400$, Adjusted $R$ Square $=.388, p<.001$.

$* p>.05$ 
Lee, J. (2020). Mediating role of resilience between successful aging and acculturation among older Korean immigrants In the United States. Advances in Social Sciences Research Journal, 7(5) 117-133.

Interestingly, the results showed there was no statistically significant relationship $(B=.73, \beta=.119$, $t[99]=1.495, p>.05$ ) between successful aging and acculturation although the results from the Correlational Analysis, and Simple Linear Analysis showed that both resilience and acculturation were significant in the relationships with successful aging. Additional tests were needed to confirm the results. At that time, the mediating effects of resilience between successful aging and acculturation were assumed because resilience might be able to control the variable of acculturation, and acculturation may be statistically significant only under the variable of resilience (Baron \& Kenny, 1986).

\section{Mediation Analysis}

It was hypothesized and partially confirmed via Simple Linear Regression Analysis that higher acculturation scores were indicative of higher level of successful aging. However, according to the result of Multiple Regression Analysis, there was no statistically significant between the relationship of acculturation and successful aging (See the Table 2-3). Resilience was a positive predictor of successful aging, and a series of Regression Analyses were conducted to find out the mediating effects of resilience between the relationship of successful aging and acculturation. Finally, it was found that resilience mediated the relationship between acculturation and successful aging.

Table 6:Steps for Mediating Effect of Resilience

\begin{tabular}{|c|c|c|c|c|c|c|c|}
\hline Regressions & Predictors & $\mathbf{R}^{2}$ & $\begin{array}{c}\text { Adjusted } \\
\mathbf{R}^{2}\end{array}$ & $F$ & $\beta$ & $t$ & Sig. \\
\hline $\begin{array}{c}\text { Step 1: } \\
\text { Predicting } \\
\text { Successful } \\
\text { Aging }\end{array}$ & Acculturation & .064 & .054 & 6.785 & .252 & 2.605 & $.011^{*}$ \\
\hline $\begin{array}{c}\text { Step 2: } \\
\text { Predicting } \\
\text { Resilience }\end{array}$ & Acculturation & .050 & .040 & 5.231 & .223 & 2.287 & $.024 *$ \\
\hline $\begin{array}{c}\text { Step 3: } \\
\text { Predicting } \\
\text { Successful } \\
\text { Aging }\end{array}$ & Resilience & .386 & .380 & 62.970 & .622 & 7.935 & $.000^{* *}$ \\
\hline $\begin{array}{c}\text { Step 4: } \\
\text { Predicting } \\
\text { Successful } \\
\text { Aging }\end{array}$ & $\begin{array}{l}\text { (1)Acculturation } \\
\text { (2) Resilience }\end{array}$ & .400 & .388 & 32.992 & $\begin{array}{l}.119 \\
.595\end{array}$ & $\begin{array}{l}1.495 \\
7.450\end{array}$ & $\begin{array}{l}.138^{* * *} \\
.000^{* *}\end{array}$ \\
\hline
\end{tabular}

Table 6 displays the steps to look for mediating effect of resilience. In Step 1 of the mediation model, the regression of Acculturation on Successful Aging, ignoring the mediator, was significant, $\beta=.252$, $t(102)=2.605, p=.011(p<.05)$. Step 2 showed that the regression of the Acculturation scores on the mediator, Resilience, was also significant, $\beta=.223, t(102)=.024, p=.024(p<.05)$. Step 3 of the mediation process showed that the mediator Resilience, controlling for the Successful Aging scores, was significant, $\beta=.622, t(102)=7.935, p=.000(p<.001)$. Step 4 of the analyses revealed that, 
controlling for the mediator Resilience, Successful Aging scores were still a significant predictor of Acculturation scores, $\beta=.595, t(102)=7.450, p=.001(p<.001)$ (Baron \& Kenny, 1986).

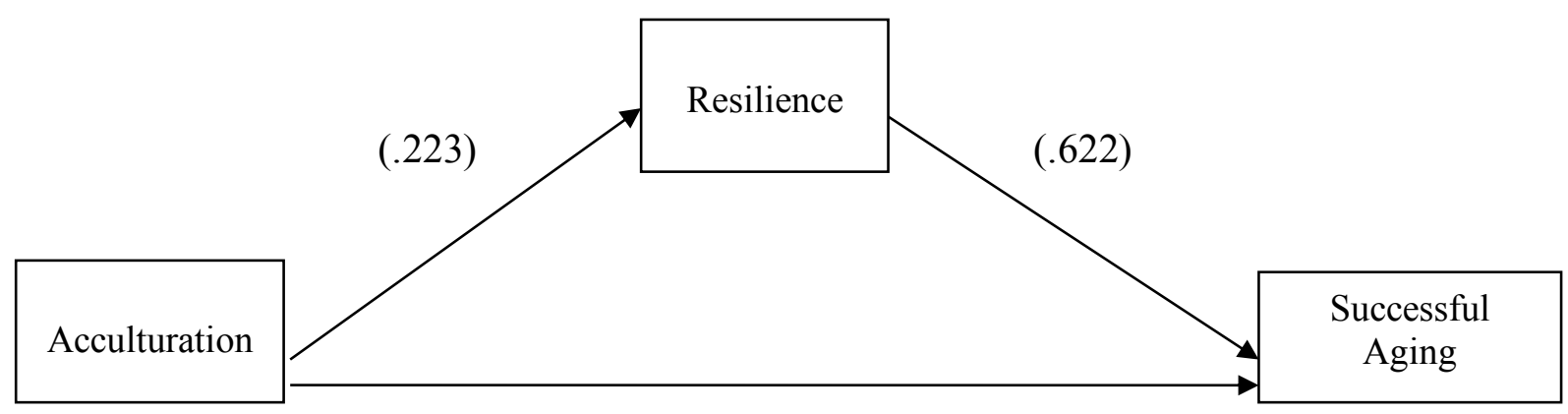

$(.595)$

Figure 1. The standardized regression coefficient $(\beta)$ for the relationship between Acculturation and Successful Aging as mediated by Resilience

Figure 1 illustrates that the standardized regression coefficient $(\beta)$ between Acculturation and Resilience was statistically significant, as was the standardized regression coefficient between Resilience and Successful Aging. The standardized indirect effect was $(.223)(.622)=.138$, and the significance of this indirect effect was tested as well. Unstandardized indirect effects were computed for each variable, and the 95\% confidence interval was computed by determining the indirect effects at the 2.5th and 97.5th percentiles. Then, it was found that the indirect effect was statistically significant (Preacher, \& Leonardelli, 2010).

In sum, a statistically positive significant relationship was found between successful aging and resilience as evidenced by the test results of Correlational Analysis, Simple Linear Regression, and Multiple Regression Analysis. In addition, a statistically positive significant relationship was found between successful aging and acculturation as evidenced by the test results of Correlational Analysis, and Simple Linear Regression Analysis; however, no statistically significant relationship was found between them according to the results of the Multiple Regression Analysis. Therefore, it was assumed and confirmed that there was a mediating effect of resilience between successful aging and acculturation because acculturation was statistically significant only under the control of resilience in the relationship with successful aging.

\section{The Mediating Role of Resilience}

It was an unexpected finding that resilience mediated the relationship between acculturation and successful aging among older Korean immigrants. As Figure 2 illustrates, it was confirmed that there was a statistically significant in mediating role of resilience between successful aging and acculturation. It was tested and confirmed that acculturation could be statistically significant under the control of mediating role of resilience between successful aging and acculturation. 


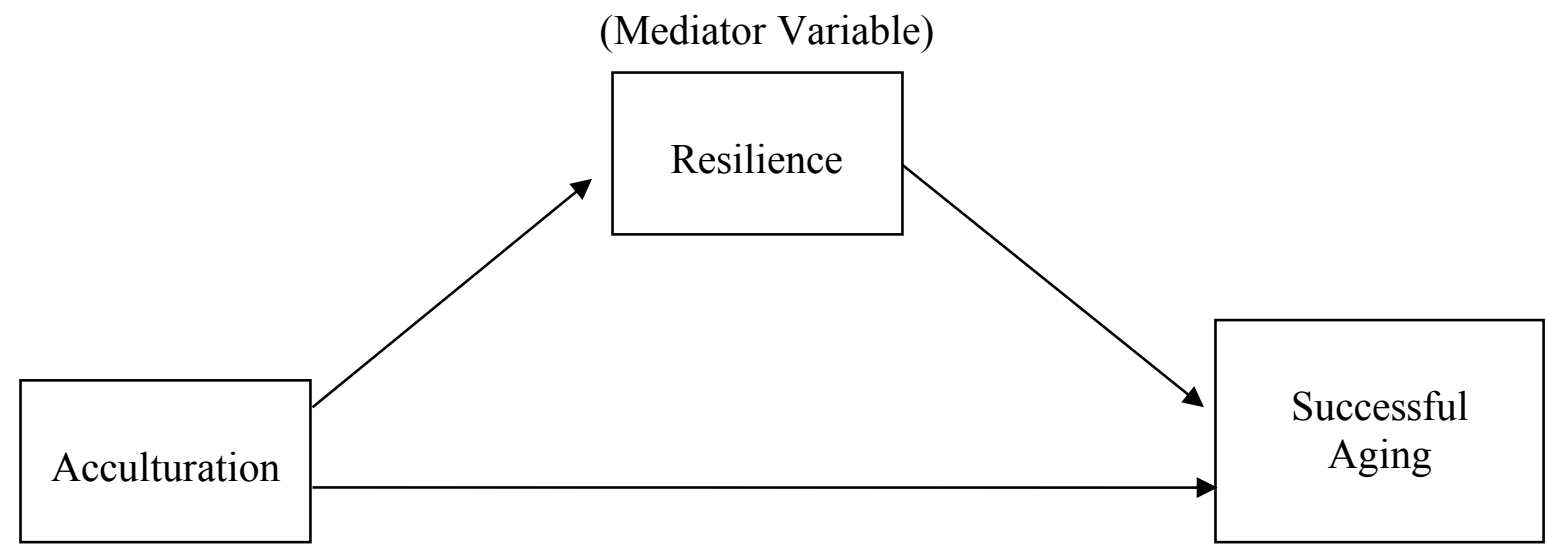

(Independent Variable)

(Dependent Variable)

\section{Figure 2. Mediating role of resilience between the relationship of successful aging and acculturation}

Mediation has been defined as a model to identify and explicate the mechanism or process that underlines an observed relationship between an independent variable and a dependent variable via the inclusion of a third explanatory variable, known as a mediator variable (Baron \& Kenny, 1986). It specifies a given cause (original predictor variable, which is independent variable) that works indirectly through a more direct cause (mediator variable) to a final effect (outcome variable, which is dependent variable). The mediator is adding to the overall variance accounted for in the model and trying to explain 'why' the dependent variable and independent variable are related (Baron \& Kenny, 1986).

In this study of successful aging among older Korean immigrants in the United States, it was found that there was a positive relationship between acculturation and successful aging throughout the test results of Correlational Analysis, and Simple Linear Regression. However, there was no statistically significant in Multiple Regression Analysis. Although there was no hypothesis regarding the mediated associations or mediated effects of resilience between successful aging and acculturation, or mediated role of acculturation between successful aging and resilience, it was assumed that the acculturation could be statistically significant only under the control of resilience. Expansively, resilience was expected to have a mediating role between successful aging and acculturation.

There was a curiosity about the relationship between acculturation and resilience, and it was tested and confirmed that there was a positive relationship between resilience and acculturation. There was no hypothesis that there was a mediating role of resilience between successful aging and acculturation or a mediating role of acculturation between successful aging and resilience, however it was assumed that resilience or acculturation could possibly influence the relationships among the variables based on SPSS results. Finally, it was tested and confirmed that acculturation was only statistically significant under the control of resilience in the relationship with successful aging. Thus, it was found that resilience was mediating between the relationship of successful aging and acculturation. 
The newly developed model in Figure 3 predicts that higher acculturation predicts higher level of successful aging. Resilience is not only a significant predictor of successful aging but also is a mediator variable that accounted for a significant amount of variance in the relationship between acculturation and successful aging. One of the major goals of this study was to examine the associations/relationships including role of two resources, acculturation and resilience as measured by the scales of acculturation and resilience. This resource, resilience completely mediated the relationship between successful aging and acculturation. Resilience appeared to provide a sense of comfort and relief to older Korean immigrants who endured physical, emotional and social limitations.

Furthermore, resilience partially increased the level of acculturation among the populations by reducing such thoughts about lack of confidence to interact with other ethnic groups, stereotypes about American culture, stress about living in America and from limited English, and pressure to become a part of the American culture. This finding highlighted the need for older Korean immigrants to enhance the ability to bounce back from their negative environments. Social workers and other practitioners for this population also need to conduct needs assessment on the dynamics of the resilience support system such as family/social support, and to find ways to increase chances of resilience practices such as community aging programs and spiritual activities.

Resilience also helped to partially reduce the uncomfortable feelings about Korean culture, stereotypes about Korean culture, and feelings of being looked down due to Korean customs. Interestingly, a certain type of protective factors in the scale of Resilience such as finding meaning and purpose in life appeared to reduce a negative factor in acculturation and in a sense enabled older Korean immigrants to turn into more adaptive ways to cope with acculturative stressors. This finding also has important social work implications in that it highlights the need for social workers and other related practitioners to assess resilience assessment, in addition to designing interventions that promote resilience well-being.

The Resilience scale measured a variety of aspects to overcome their negative environments so that they could find meaning and purpose in their life (Hooyman \& Kiyak, 2011). One of the subscales in this measure assessed the value individual coping mechanism such as the importance of seeing bright side of the problem, having a sense of humor, recalling the experience of overcoming obstacles. This dimension of individual coping skills is possibly one of the most important factors to become successful agers in terms of their perception toward successful aging such as positive attitude to current life and acceptance of current life status.

If older Korean immigrants find meaning and purpose in life from increasing individual coping skills, and can meet the supports of children, family and other loved ones, their feelings of aging successful will be the maximized. It is because there was a great connectedness with successful aging and their values in children, family, community, and other loved ones around them. If they are dissatisfied with the level of support from children, family and other significant ones in their community, their feelings of isolation or abandonment will emerge. Perhaps, a lack of connections among the older Korean immigrants with their children, family, and other significant ones in their community and limited physical and emotional support from them will create serious problems such as social isolation and abandonment that can be linked with depressive symptoms. This explanation can provide some rationale why older adults who lacked physical and emotional 
Lee, J. (2020). Mediating role of resilience between successful aging and acculturation among older Korean immigrants In the United States. Advances in Social Sciences Research Journal, 7(5) 117-133.

support reported more depressive symptoms and unmet needs. Of course, further research would be needed to explore other possible mechanisms and pathways in predicting older adults' resilience.

\section{LIMITATIONS}

The cross-sectional design was a limitation. In the case of Rowe and Kahn's study of successful aging, it was a longitudinal study following up over 2000 study participants for more than 10 years. This study of successful aging among older Korean immigrants was a one-time survey that could not provide information about changes in attitude over time. Multiple observations at multiple times might be able to produce different findings that could contribute to better understanding about successful aging among the population.

Next, the survey instruments that were used for this study were originally developed in English. Those scales had several different language versions including Korean, but there was no way to check if the Korean version was culturally validated for Korean immigrant settings. In addition, although efforts to ensure language equivalence were made via translation and back translation, cultural validation including culturally sensitive interpretation was required, especially when the responses of the open-ended questions asking about their perceptions toward successful aging. All the responses of the questions were Korean, and when they were translated and back translated to English, there were limitations when choosing appropriate words and expressions. For example, when they were emphasizing on the relationship with others, they used various expressions, more than six or seven numbers of words to describe the relations among others such as relations with older people, younger people, or same aged people in Korean, but when those word were interpreted to English, 'interactions' or 'socialization' were the most appropriate words. It was also same when they expressed the positive attitude to their current life and accepting their situations. They used various expressions in Korean to describe their attitudes to current life, but when they were translated to English, 'positive' was the best word. The limitation was it was somewhat broad and did not fully represent those in Korean.

Last, this study's non-probability sampling method was a limitation in the generalizability of findings to the population at large (Rubin \& Babbie, 2010). Again, study was hard to represent all the older Korean immigrants in the United States because all the study participants resided in the Korea Town in L.A, CA. Older Korean immigrants in different regions with different background, environment, perspectives, and socioeconomic status might be able to respond differently to the questionnaires that could yield different results of the study.

\section{CONCLUSION}

This study examined the relationship between successful aging and resilience, and successful aging and acculturation among older Korean immigrants in the United States. It has identified significant factors influencing the population's aging successfully, and the findings of this study indicated that there were positively significant relationships among successful aging, resilience, and acculturation.

Overall respondents were likely to have a positive attitude toward their immigrant life and were optimistic toward their successful aging although most of them were placed under the poverty line and having any types of physical or cognitive deteriorations. One of the important finding was that the participants considered the positive attitude toward their current life status and acceptance of 
it as important factors and predictors to determine successful aging. Another important finding was the mediating role of resilience between the relationship of successful aging and acculturation.

The study addressed that current rising issues in aging study such as positive aging (successful aging and productive aging), and meaningful life in baby boomer generation's retirement years were somewhat toward to mainstream populations. The study also intended to raise concerns about successful aging among older minorities and immigrants because they have been marginalized by aging research studies. The findings of this study suggest the need for more attention to the populations of older immigrants/minorities, and innovations of community aging programs for those populations. For these innovations, modernizations of aging policies should be preceded, and for the modernizations of aging policies, rigorous research activities should be accompanied.

Social workers need to be a frontier for these innovations and will need to be cognizant that community aging services and interventions for successful aging should be ethically and culturally appropriate and in alignment with the needs of new generation of older populations who have various perspectives, experiences, and uniqueness toward their life. Social work practitioners, researchers, and educators' mission to deliver compassionate care aimed at promoting resiliency, enhancing older populations' quality of life, and fostering the dignity and worth of both older adults and their family will become more important in years to come.

\section{References:}

Adams, K. B., Leibbrandt, S., \& Moon, H. (2011). A critical review of the literature on social and leisure activity and wellbeing in later life, Ageing Society, 31, 683-701.

Asian American Federation. (2003). Growing older: Asian American Elders in New York City. New York: Asian American Federation.

Atchley, R. C. (1989). A continuity theory of normal aging, The Gerontologist, 29(2), 183-190.

Baltes, P. B., \& Baltes, M. M. (1990). Psychological perspectives on successful aging: The model of selective optimization with compensation. In P. B. Baltes \& M. M. Baltes (Eds.), Successful aging: Perspectives from the behavioral sciences. Cambridge, MA: Cambridge University Press.

Berry, J. W. (2003). Conceptual approaches to acculturation. American Psychological Association, 27, 17-37.

Cernin, P. A. (2009). A comparison of self-rated and objectively measured successful aging constructs in an urban sample of African American older adults. The Journal of Aging and Mental Health, 34(2), 89-102.

Choi, J. B., \& Thomas, M. (2009). Predictive factors of acculturation attitudes and social support among Asian immigrants in the USA. International Journal of Social Welfare. 18, 76-84.

Chung, S. \& Park, S. (2008). Successful aging among low-income older people in South Korea. Aging and Society, 28, 1061-1074.

Creswell, J. (2009). Research design: Qualitative, quantitative, and mixed methods approaches. Thousand Oaks, CA: SAGE Publications, Inc.

Cumming, E., \& Henry, W. E. (1961). Growing old. New York, NY. Basic.

Depp, C. A., \& Jeste, D.V. (2006). Definitions and predictors of successful aging: A comprehensive review of larger quantitative studies. American Journal of Geriatric Psychiatry, 14 (1), 6-20.

Dillaway, H. E. \& Byrnes, M. (2009). Reconsidering successful aging: A call for renewed and expanded academic critiques and conceptualization, Journal of Applied gerontology,28(6), 702-722. 
Lee, J. (2020). Mediating role of resilience between successful aging and acculturation among older Korean immigrants In the United States. Advances in Social Sciences Research Journal, 7(5) 117-133.

Ellison, C. W. (1983). Spiritual well-being: conceptualization and measurement. Journal of Psychology and Theology, 11(4), 330-340.

Ferri, C., James, I., \& Pruchno, R. (2009). Successful aging: definition and subjective assessment according to older adults, Clinical gerontologist, 32(4), 379-388.

Han, H., Kim, M., Lee, H. B., Pistulka, G., \& Kim, K.B. (2007). Correlates of depression in the Korean American elderly: Focusing on personal resources of social support. Journal of Cross-Cultural Gerontology, 22, 115-127.

Hooyman, N. R., \& Kiyak, H. A. (2011). The resilience of elders of color. Social Gerontology (9th ed.). Boston, MA: Allyn \& Bacon.

Jang, Y., Kim, G., \& Chiriboga, D. A. (2006). Health perception and depressive symptoms among older Korean Americans. Journal of Cross-Cultural Gerontology, 21, 91-102.

Knight, T., \& Ricciardelli, L.A. (2003). Successful aging: Perceptions of adults aged between 70 and 101 years. International Journal of Aging and Human Development, 56, (3) 223-245.

Larson, E. B., \& Phelen, E. A. (2002). "Successful aging”: Where next? Journal of the American Geriatrics Society, 50, 1306-1308.

Lee, K. H., \& Hwang, M. J., (2014). Private Religious Practice, Spiritual Coping, Social Support, and Health Status among Older Korean Adult Immigrants. Social Work in Public Health, $\quad$ 29(5), p428-443.

Lee, Y. M. (2007). The immigration experience among elderly Korean immigrants. Journal of Psychiatric and Mental Health Nursing. 14, 403-410.

Liang, J., \& Luo, B. (2012). Toward a discourse shift in social gerontology: From successful aging to harmonious aging, Journal of Aging Studies, 26(3), 327-334.

Li, C., Wu, W., Jin, H., Zhang, X., Hue, H., He, Y., Xiao, S., Jeste, D., \& Zhang, M. (2006). Successful aging in Shanghai, China: Definition, distribution and related factors. International Psychogeriatrics, 18(3), 551-563. Psychiatric and Mental Health Nursing. 14, 403-410.

Maki, A. (2005). Relationship between spirituality and successful aging among older minority women, Dissertation Abstracts International, 66-07, 3975B.

McLaughlin, S. J., Connell, C. M., Heeringa, S. G., Li, L. W., \& Roberts, S. (2009). Successful aging in the United States: Prevalence estimates from a national sample of older adults. Journal of Gerontology: Social Sciences, 65B(2), 216-226.

Min, J. W. \& Moon, A. (2006). Older Asian Americans, Handbook of Social Work in Health and Aging. Oxford University Press, 2006.

Nagalingam, J. (2007). Understanding successful aging: A study of older Indian adults in Singapore, Care Management Journal, 8(1), 18-25.

Oh, K. K., Zhou, Q., Kreps, G. \& Kim, W. (2014). The influences of immigration on health information seeking behaviors among Korean Americans and Native Koreans, Health Education \& Behavior 41(2) 173-185.

Phelen, E.A., Anderson, L.A., LaCroix, A.Z., \& Larson, E.B. (2004). Older adults views of successful aging: How do they compare with researchers' definitions? Journal of the American Geriatrics Society, 52, 211-216.

Rowe, J.W, \& Kahn, R. L. (1997). Successful aging. The Gerontologist, 37, 433-440.

Strawbridge, W.J., Wallhagen, M.I., \& Cohen, R.D. (2002). Successful aging and well-being: Self-rated compared with Rowe and Kahn. The Gerontologist, 42,(6), 727-733.

Tan, J., Ward, L., Ziaian, T. (2011). Comparing definitions of successful ageing: The case of Anglo-and Chinese Australians, Journal of Applied Psychology, 7(1), 15-21.

Tate, R., Lah, L., \& Cuddy, T. E. (2003). Definition of successful aging by elderly Canadian males: The manitoba followup study, The Gerontologist, 43(5), 735-744.

US Census Bureau. (2017). The 2017 Statistical Abstract. Retrieved from http://www.census.gov/compendia/statab/cats/population.html. 
Victor, B. (2004). Successful aging and the Chinese-American elder, Dissertation Abstracts International, 65-12, 4434A. 